\title{
Prognostic Value of Admission Mean Corpuscular Volume for Major Adverse Cardiovascular Events following Stent Implantation in Nondiabetic and Diabetic Patients with Acute Coronary Syndrome
}

\author{
Lele Cheng, ${ }^{1}$ Lisha Zhang, ${ }^{1}$ Junhui Liu, ${ }^{2}$ Wenyuan Li, ${ }^{1}$ Xiaofang Bai, ${ }^{1}$ Ruifeng Li, ${ }^{1}$ Bolin Li, \\ Lijun Wang, ${ }^{1,3}$ Juan Zhou, ${ }^{1,3}$ Yue $W u \mathbb{D}^{1,3}$ and Zuyi Yuan $\mathbb{D}^{1,3,4}$ \\ ${ }^{1}$ Department of Cardiovascular Medicine, The First Affiliated Hospital, Xi'an Jiaotong University, Xi'an, Shaanxi, China \\ ${ }^{2}$ Department of Clinical Laboratory, The First Affiliated Hospital, Xi'an Jiaotong University, Xi'an, Shaanxi, China \\ ${ }^{3}$ Key Laboratory of Molecular Cardiology, Shaanxi Province, Xi'an, Shaanxi, China \\ ${ }^{4}$ Key Laboratory of Environment and Genes Related to Diseases, Ministry of Education, Xi'an, Shaanxi, China \\ Correspondence should be addressed to Yue Wu; yue.wu@xjtu.edu.cn and Zuyi Yuan; zuyiyuanxjtu@126.com
}

Received 6 October 2019; Revised 20 June 2020; Accepted 29 June 2020; Published 18 July 2020

Academic Editor: Wen-Jun Tu

Copyright (c) 2020 Lele Cheng et al. This is an open access article distributed under the Creative Commons Attribution License, which permits unrestricted use, distribution, and reproduction in any medium, provided the original work is properly cited.

\begin{abstract}
Background. One of the key concerns of the clinician is to identify and manage risk factors for major adverse cardiovascular events (MACEs) in nondiabetic and diabetic patients with acute coronary syndrome (ACS) undergoing stent implantation. Mean corpuscular volume (MCV) is a marker of erythrocyte size and activity and is associated with prognosis of cardiovascular disease. However, the role of admission MCV in predicting MACEs following stent implantation in diabetes mellitus (DM), non-DM, or whole patients with ACS remains largely unknown. Methods and Results. A total of 437 ACS patients undergoing stent implantation, including 294 non-DM (59.08 \pm 10.24 years) and 143 DM (63.02 \pm 9.92 years), were analyzed. Admission MCV was higher in non-DM than DM patients. During a median of 31.93 months follow-up, Kaplan-Meier curve demonstrated that higher admission MCV level was significantly associated with increased MACEs in whole and non-DM, but not in DM patients. In Cox regression analysis, the highest MCV tertile was associated with higher MACEs in whole ([HR] $1.870,95 \%$ CI 1.113-3.144, $P=0.018$ ), especially those non-DM ([HR] 2.089, 95\% CI 1.077-4.501, $P=0.029)$ patients after adjustment of several cardiovascular risk factors. MCV did not predict MACEs in DM patients. During landmark analysis, admission MCV showed better predictive value for MACEs in the first 32 months of follow-up than in the subsequent period. Finally, the receiver operating characteristic (ROC) curve was conducted to confirmed the value of admission MCV within 32 months. Conclusion. In patients with ACS, elevated admission MCV is an important and independent predictor for MACEs following stent implantation, especially amongst those without DM even after adjusting for lifestyle and clinical risk factors. However, as the follow-up period increased, the admission MCV lost its ability to predict MACEs.
\end{abstract}

\section{Introduction}

Coronary artery disease (CAD) is caused by atherosclerosis accompanied by stenosis or lumen obstruction, resulting in myocardial ischemia, hypoxia, and necrosis [1-3]. It has become an important threat to human life and health, especially in middle- and low-income countries $[4,5]$. Acute cor- onary syndrome (ACS) is a serious type of CAD that can lead to various adverse outcomes $[6,7]$. Percutaneous coronary intervention (PCI) is a common revascularization strategy in the management of ACS due to its safety profile, low complication rates, symptom relief, and survival benefits $[8,9]$. Although significant achievements have been made in the treatment, major adverse cardiovascular events (MACEs) 


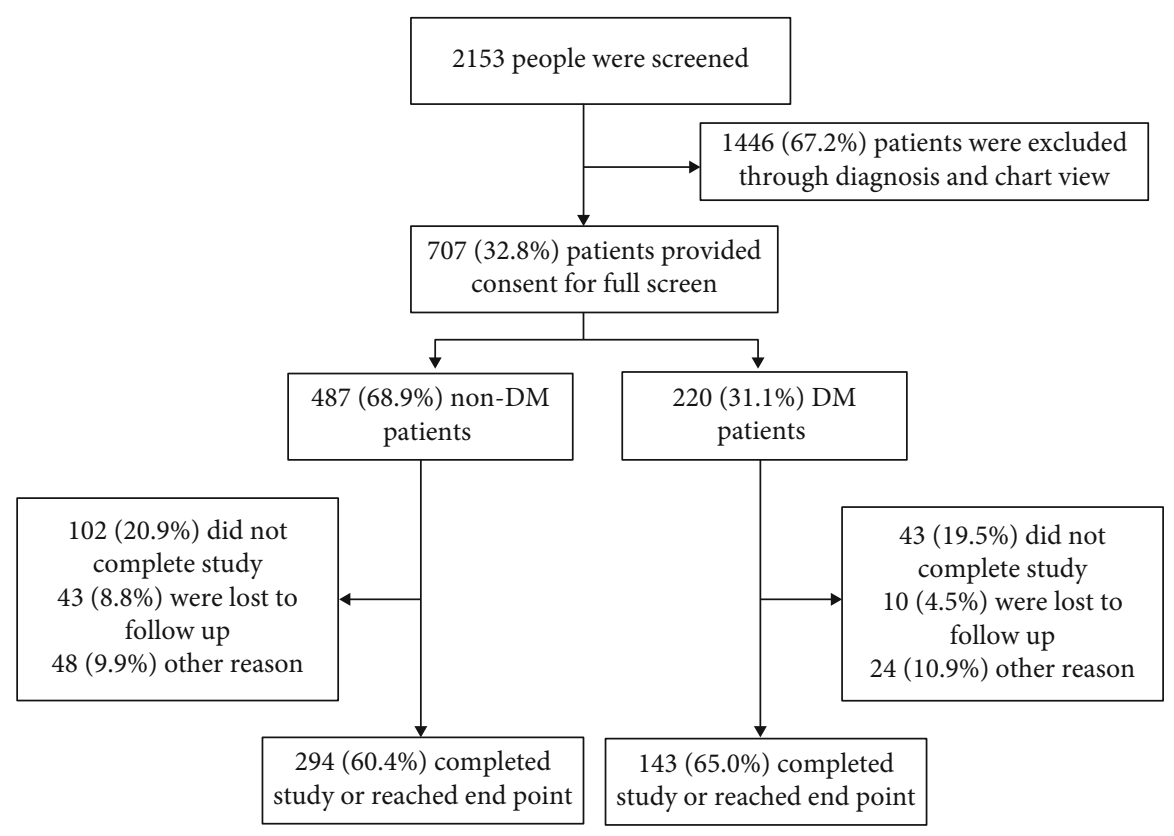

FIGURE 1: Study flow for the present analysis.

remains a frequent and severe complication following stent implantation $[10,11]$.

The incidence of diabetes mellitus (DM), a metabolic disorder characterized by hyperglycemia, is a major and important risk factor for cardiovascular events including ACS undergoing PCI $[12,13]$. Sustained hyperglycemia is shown to exacerbate cardiac damage through changes of erythrocyte [14]. Mean corpuscular volume (MCV) is the measure of the average size of erythrocytes, which is closely related to erythrocyte disorders $[15,16]$. It is reduced in diabetes, probably because iron deficiency anemia is common in people with diabetes $[17,18]$. Studies demonstrate that MCV is associated with the prognosis of several diseases, including peripheral arterial disease, CAD, and cerebral ischemic stroke [19-23]. The possible mechanism is that the increase of MCV impairs the antioxidant capacity of erythrocytes and decreases endothelial function, both of which promote the development of atherosclerosis [24]. In addition, high MCV would be related to a hindered flow of larger, less flexible erythrocytes through microcirculation, which might contribute to myocardial ischemia $[25,26]$. However, the role and difference of MCV in predict MACEs between nondiabetic and diabetic populations with ACS undergoing PCI still largely unknown. Therefore, exploring the relationship between MCV level and adverse outcomes following stent implantation in nondiabetic and diabetic patients with ACS is of great clinical value.

In this context, the present study was performed to explore the value of admission MCV for prognosis of MACEs in ACS patients, with or without DM in China.

\section{Materials and Methods}

2.1. Study Population. The present study was carried out in the First Affiliated Hospital of Medical College of Xi'an Jiaotong University from January 2013 to February 2014. The inclusion criteria were confirmed admission diagnosis of
ACS including ST-elevation myocardial infarction (STEMI), non-ST-segment elevation myocardial infarction (NSTEMI), and unstable angina pectoris (UA), successful treatment by stent implantation, without diabetic ketosis or nonketotic hyperosmolar coma. The exclusion criteria were patients with malignant tumors, severe renal and liver diseases, pregnant patients, patients with autoimmune diseases or blood disorders, severe cerebrovascular diseases, and prior history of surgical treatment within 2 weeks of this study, and data were not available for the MCV levels. Qualitative and quantitative coronary angiographic analyses were carried out according to standard methods. PCI was performed using standard techniques. All patients were given loading doses of aspirin $(300 \mathrm{mg})$, clopidogrel $(600 \mathrm{mg})$, and unfractionated heparin $(100 \mathrm{U} / \mathrm{kg})$ before coronary intervention. The treatment strategy, stenting techniques, and use of glycoprotein IIb/IIIa receptor inhibitors or intravascular ultrasound used at the discretion of the operator during the PCI procedures. Drug-eluting stents were used (Medtronic, Inc.). The success of PCI is defined as previously described [27, 28]. Written informed consent was obtained from all study participants, approved by ethic committee approval at the First Affiliated Hospital of Xi'an Jiaotong University.

The estimation of sample size was performed using PASS software (version 11.0) [29]. A sample size of 311 achieves $90 \%$ power to detect an effect size of 0.202 using a 2 degrees of freedom chi-square test with a significance level of 0.050 .

2.2. Demographic and Clinical Data. Main demographic data, cardiovascular risk factors, and cardiovascular drugs received were obtained from medical records. A diagnosis of DM was based on the criteria by the American Diabetes Association [30]. Hypertension was defined as resting blood pressure $\geq 140 / 90 \mathrm{mmHg}$ at two different visits, and having a history of hypertension or receiving hypertension drugs. Previous 
TABLE 1: Characteristics of whole ACS patients undergoing stent implantation and with or without DM.

\begin{tabular}{|c|c|c|c|c|}
\hline Variable & Whole $(n=437)$ & Non-DM $(n=294)$ & $\mathrm{DM}(n=143)$ & $P$ value \\
\hline Mean corpuscular volume, $\mathrm{fl}$ & $93.30(90.25-96.35)$ & $94.05(90.80-97.50)$ & $91.30(88.40-94.60)$ & $<0.001$ \\
\hline Age, years & $60.35 \pm 10.27$ & $59.08 \pm 10.24$ & $63.02 \pm 9.92$ & 0.002 \\
\hline \multicolumn{5}{|l|}{ Sex } \\
\hline Male, \% & $340(77.8)$ & $237(80.6)$ & $103(72.3)$ & 0.060 \\
\hline Body mass index, $\mathrm{kg} / \mathrm{m}^{2}$ & $25.00 \pm 3.26$ & $25.05 \pm 3.35$ & $24.99 \pm 3.23$ & 0.558 \\
\hline Smoking, \% & $246(56.3)$ & $170(57.8)$ & $76(53.1)$ & 0.411 \\
\hline Past MI, \% & $67(15.3)$ & $50(17.0)$ & $17(11.9)$ & 0.211 \\
\hline Past PCI or CABG, \% & $85(19.5)$ & $59(20.1)$ & $26(18.2)$ & 0.735 \\
\hline Hypertension, \% & $227(51.9)$ & $148(50.3)$ & $79(55.2)$ & 0.389 \\
\hline Family history, \% & $176(40.1)$ & $125(42.5)$ & $51(35.7)$ & 0.205 \\
\hline Diastolic blood pressure, $\mathrm{mmHg}$ & $80(70-84)$ & $80(70-84)$ & $80(70-84)$ & 0.934 \\
\hline Systolic blood pressure, $\mathrm{mmHg}$ & $120(114-140)$ & $120(112-140)$ & $127(114-140)$ & 0.281 \\
\hline Heart rate, bmp & $69(61-78)$ & $69(61-78)$ & $70(62-77)$ & 0.982 \\
\hline Hemoglobin A1c, \% & $6.00(5.60-6.70)$ & $5.70(5.50-6.00)$ & $7.40(6.60-8.80)$ & $<0.001$ \\
\hline Left ventricle ejection fraction, $\%$ & $61(49-68)$ & $62(49-68)$ & $59(45-68)$ & 0.117 \\
\hline Hemoglobin, g/L & $140.00(128.00-151.00)$ & $141.00(129.50-151.00)$ & $137.00(123.00-149.00)$ & 0.032 \\
\hline Platelet, $10^{3}$ cells $/ \mathrm{dL}$ & $181.00(147.00-217.00)$ & $183.50(149.70-224.00)$ & $176.00(140.00-205.00)$ & 0.055 \\
\hline Creatinine, $\mathrm{mg} / \mathrm{dL}$ & $66.71(58.27-77.37)$ & $67.38(58.66-77.40)$ & $64.74(56.04-77.37)$ & 0.527 \\
\hline Triglycerides, mmol/L & $1.42(1.08-1.96)$ & $1.42(1.09-1.94)$ & $1.42(1.00-1.98)$ & 0.854 \\
\hline High-density lipoprotein, mmol/L & $0.87(0.76-1.03)$ & $0.89(0.76-1.06)$ & $0.84(0.74-0.99)$ & 0.134 \\
\hline Low-density lipoprotein, $\mathrm{mmol} / \mathrm{L}$ & $2.13(1.63-2.68)$ & $2.18(1.65-2.73)$ & $2.04(1.56-2.59)$ & 0.128 \\
\hline Apolipoprotein A1, g/L & $1.05(0.96-1.19)$ & $1.05(0.96-1.19)$ & $1.05(0.94-1.19$ & 0.678 \\
\hline High-sensitivity C-reactive protein, mg/L & $1.40(0.74-3.50)$ & $1.46(0.76-3.61)$ & $1.19(0.64-2.99)$ & 0.375 \\
\hline Creatine kinase isoenzymes $\mathrm{MB}, \mathrm{U} / \mathrm{L}$ & $15.70(11.44-32.33)$ & $15.70(11.30-35.60)$ & $15.75(12.10-26.50)$ & 0.864 \\
\hline Pro-B-type natriuretic peptide, $\mathrm{pg} / \mathrm{mL}$ & $377.45(122.86-1008.50)$ & $377.45(122.18-1003.50)$ & $377.80(135.86-1063.25)$ & 0.924 \\
\hline \multicolumn{5}{|l|}{ Medicine at discharge, $\%$} \\
\hline Aspirin & $433(99.1)$ & $291(99.0)$ & $142(99.3)$ & a \\
\hline Clopidogrel & $425(97.3)$ & $285(96.9)$ & $140(97.9)$ & 0.679 \\
\hline ACEI/ARB & $398(91.1)$ & $269(91.5)$ & $129(90.2)$ & 0.792 \\
\hline Calcium channel blocker & $95(21.7)$ & $60(20.4)$ & $35(24.5)$ & 0.399 \\
\hline$\beta$-Blockers & $385(88.1)$ & $262(89.1)$ & $123(86.0)$ & 0.434 \\
\hline Statin & $429(98.2)$ & $289(98.3)$ & $140(97.9)$ & a \\
\hline
\end{tabular}

Data are presented as mean $\pm \mathrm{SD}$, median $(\mathrm{IQR})$ or number $(\%)$, "a" represents $P$ value $=1$. ACS: acute coronary syndrome; DM: diabetes mellitus; MI: myocardial infarction; PCI or CABG: past percutaneous coronary intervention or coronary artery bypass grafting; ACEI: angiotensin-converting enzyme inhibition; ARB: angiotensin receptor blocker.

MI was based on a history of acute MI (AMI) or with signs of an infarction outside the area of the index infarction.

2.3. Biochemical Measurements. Peripheral blood was sampled from patients within $24 \mathrm{~h}$ of admission prior to stent implantation. Triglycerides (TG), low-density lipoprotein cholesterol (LDL), high-sensitivity C-reactive protein (hsCRP), creatinine, Hemoglobin Alc (HbAlc), and pro-Btype natriuretic peptide (pro-BNP) were assayed using the Cobas Integra automated chemistry analyzer (Roche Cobas Integra 400 Plus, Roche Diagnostics, USA). High-sensitivity $\mathrm{C}$-reactive protein was assessed using enzyme-linked immunosorbent assay according to the manufacturer's instructions (EIA-3954, DRG International Inc., Springfield Township, USA). Hemoglobin (HGB), MCV, and platelet counts were measured using the Sysmex KX 4000i haematology analyzer (Sysmex Corporation, Kobe, Japan) by standard biochemical techniques. The patients were further divided into 3 groups according to tertiles of MCV, and the reference MCV interval of our laboratory was 82-100 fL. Echocardiographs were performed on admission by experienced echo cardiologists, and systolic function was expressed as the ejection fraction (EF), which was calculated using Simpson's method.

2.4. Outcomes and Follow-Up. MACEs were defined as composite endpoints, including all-cause death, nonfatal AMI, urgent coronary revascularization, UA, and stroke [31]. All patients were followed up by interview or telephone in our hospital, and the end of follow-up was the date of the first MACEs occurrence. 
TABLE 2: Clinical characteristics stratified by MCV tertiles.

\begin{tabular}{|c|c|c|c|c|}
\hline \multirow[b]{2}{*}{ Variable } & \multicolumn{4}{|c|}{ Mean corpuscular volume, $\mathrm{fl}$} \\
\hline & $\begin{array}{c}\text { MCV-L (64.6-91.0) } \\
\quad N=146\end{array}$ & $\begin{array}{c}\text { MCV-M (91.1-95.3) } \\
N=145\end{array}$ & $\begin{array}{c}\text { MCV-H (95.4-113.3) } \\
N=146\end{array}$ & $P$ value \\
\hline Mean corpuscular volume, $\mathrm{fl}$ & $88.6(86.5-90.25)$ & $93.30(91.90-94.20)$ & $98.00(96.30-100.55)$ & $<0.001$ \\
\hline Age, year & $59.24 \pm 10.6$ & $60.43 \pm 9.7$ & $61.36 \pm 10.4$ & 0.210 \\
\hline \multicolumn{5}{|l|}{ Sex } \\
\hline Male, $\%$ & $105(74.0)$ & $115(79.3)$ & $120(80.1)$ & 0.183 \\
\hline Body mass index, $\mathrm{kg} / \mathrm{m}^{2}$ & $25.59 \pm 3.09$ & $24.78 \pm 3.36$ & $24.66 \pm 3.26$ & 0.064 \\
\hline Smoking, \% & $85(58.2)$ & $71(49.0)$ & $90(65.8)$ & 0.079 \\
\hline Past MI, \% & $23(15.8)$ & $23(15.9)$ & $22(15.1)$ & 0.980 \\
\hline Past PCI or CABG, $\%$ & $31(21.2)$ & $30(20.7)$ & $25(17.1)$ & 0.631 \\
\hline Hypertension, \% & $84(57.5)$ & $75(51.7)$ & $69(47.3)$ & 0.212 \\
\hline Diabetes, \% & $67(45.9)$ & $47(32.4)$ & $29(19.9)$ & $<0.001$ \\
\hline Family history, \% & $59(40.4)$ & $52(35.9)$ & $65(44.5)$ & 0.322 \\
\hline Diastolic blood pressure, $\mathrm{mmHg}$ & $80(70-90)$ & $80(70-85)$ & $80(70-86)$ & 0.154 \\
\hline Systolic blood pressure, $\mathrm{mmHg}$ & $120(118-140)$ & $120(110-140)$ & $125(114-140)$ & 0.811 \\
\hline Heart rate, bmp & $70(62-76)$ & $69(61-77)$ & $67(61-78)$ & 0.764 \\
\hline Hemoglobin A1c, \% & $6.15(5.70-7.65)$ & $6.05(5.62-6.50)$ & $5.80(5.50-6.20)$ & $<0.001$ \\
\hline Left ventricle ejection fraction, $\%$ & $62(48-69)$ & $62(47-68)$ & $61(49-68)$ & 0.873 \\
\hline Hemoglobin, g/L & $139.00(125.50-150.50)$ & $140.00(130.00-152.00)$ & $141.00(127.00-151.00)$ & 0.846 \\
\hline Platelet, $10^{3}$ cells $/ \mathrm{dL}$ & $186.00(155.50-219.00)$ & $181.00(143.75-218.00)$ & $172.00(137.00-214.00)$ & 0.230 \\
\hline Creatinine, $\mathrm{mg} / \mathrm{dL}$ & $66.43(57.35-76.69)$ & $66.32(57.26-75.33)$ & $78.81(60.05-79.60)$ & 0.174 \\
\hline Triglycerides, $\mathrm{mmol} / \mathrm{L}$ & $1.49(1.11-2.06)$ & $1.41(1.09-1.96)$ & $1.41(0.98-1.80)$ & 0.887 \\
\hline High-density lipoprotein, mmol/L & $0.87(0.77-1.03)$ & $0.89(0.76-1.28)$ & $0.87(0.74-1.07)$ & 0.670 \\
\hline Low-density lipoprotein, $\mathrm{mmol} / \mathrm{L}$ & $2.14(1.67-2.73)$ & $2.19(1.74-2.63)$ & $2.02(1.46-2.73)$ & 0.336 \\
\hline High-sensitivity C-reactive protein, mg/L & $1.53(0.74-3.63)$ & $1.16(0.69-3.00)$ & $1.38(0.74-3.78)$ & 0.423 \\
\hline Creatine kinase isoenzymes $\mathrm{MB}, \mathrm{U} / \mathrm{L}$ & $14.20(10.91-26.50)$ & $16.85(12.20-35.03)$ & $16.75(12.24-37.96)$ & 0.068 \\
\hline Pro-B-type natriuretic peptide, pg/ML & $368.90(116.60-886.30)$ & $403.80(171.50-1005.00)$ & $335.20(121.4-1160.00)$ & 0.724 \\
\hline Major adverse cardiovascular events, $\%$ & $42(28.8)$ & $37(25.5)$ & $61(41.8)$ & 0.007 \\
\hline Type of acute coronary syndrome, $\%$ & & & & 0.781 \\
\hline Unstable angina & $86(58.9)$ & $76(52.4)$ & $78(53.4)$ & \\
\hline ST-segment elevation myocardial infarction & $40(27.4)$ & $46(31.7)$ & $48(32.9)$ & \\
\hline Non-ST-segment elevation myocardial infarction & $20(13.7)$ & $23(15.9)$ & $20(13.7)$ & \\
\hline Lesion vessel number, \% & & & & 0.083 \\
\hline 1 & $15(10.3)$ & $27(18.6)$ & $26(17.8)$ & \\
\hline 2 & $43(29.5)$ & $48(33.1)$ & $35(24.0)$ & \\
\hline 3 & $88(60.3)$ & $70(48.3)$ & $85(58.2)$ & \\
\hline \multicolumn{5}{|l|}{ Medicine at discharge, $\%$} \\
\hline Aspirin & $146(100)$ & $145(100)$ & $142(97.3)$ & a \\
\hline Clopidogrel & $140(95.9)$ & $143(98.6)$ & $142(97.3)$ & 0.404 \\
\hline ACEI/ARB & $133(91.1)$ & $134(92.4)$ & $131(89.7)$ & 0.724 \\
\hline Calcium channel blockers & $40(27.4)$ & $24(16.4)$ & $31(21.2)$ & 0.080 \\
\hline$\beta$-Blockers & $125(85.6)$ & $126(86.9)$ & $134(91.8)$ & 0.230 \\
\hline Statin & $143(97.9)$ & $143(98.6)$ & $143(97.9)$ & $\mathrm{a}$ \\
\hline
\end{tabular}

Data are presented as mean $\pm \mathrm{SD}$, median (IQR) or number (\%); "a" represents $P$ value $=1$. MCV: mean corpuscular volume; MI: myocardial infarction; PCI or CABG: past percutaneous coronary intervention or coronary artery bypass grafting; ACEI: angiotensin-converting enzyme inhibition; ARB: angiotensin receptor blocker.

2.5. Statistical Analysis. The statistical analyses in this study were performed using SPSS for Windows 25.0 (SPSS Inc, Chicago, IL), R software (version 3.6.1), and EmpowerStats (http://www.empowerstats.com/). Continuous variables were expressed as mean $\pm \mathrm{SD}$ or median (interquartile range (IQR)) according to different distributions, and in case of 


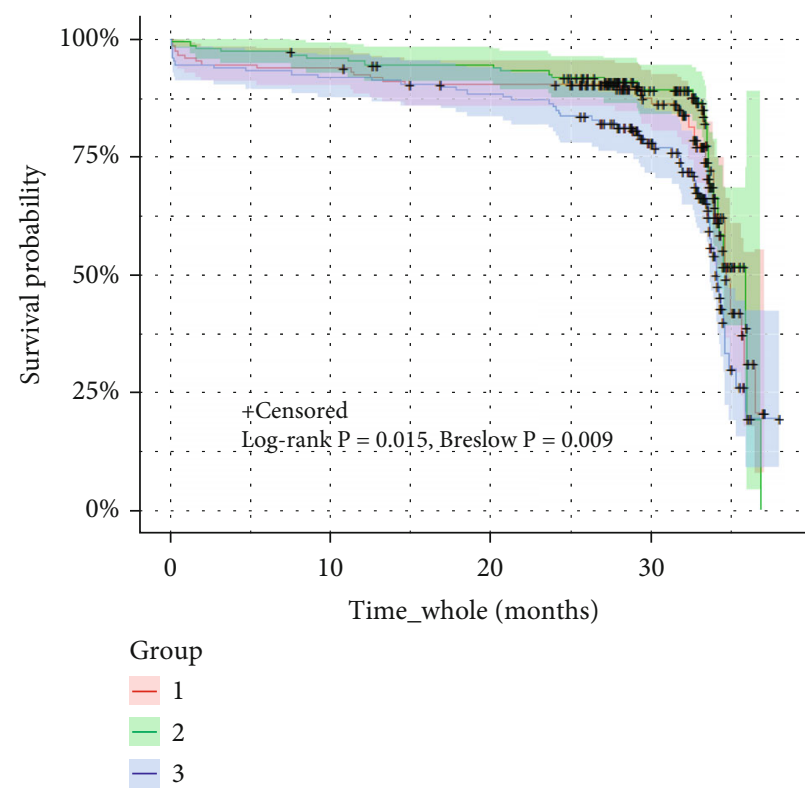

(a)

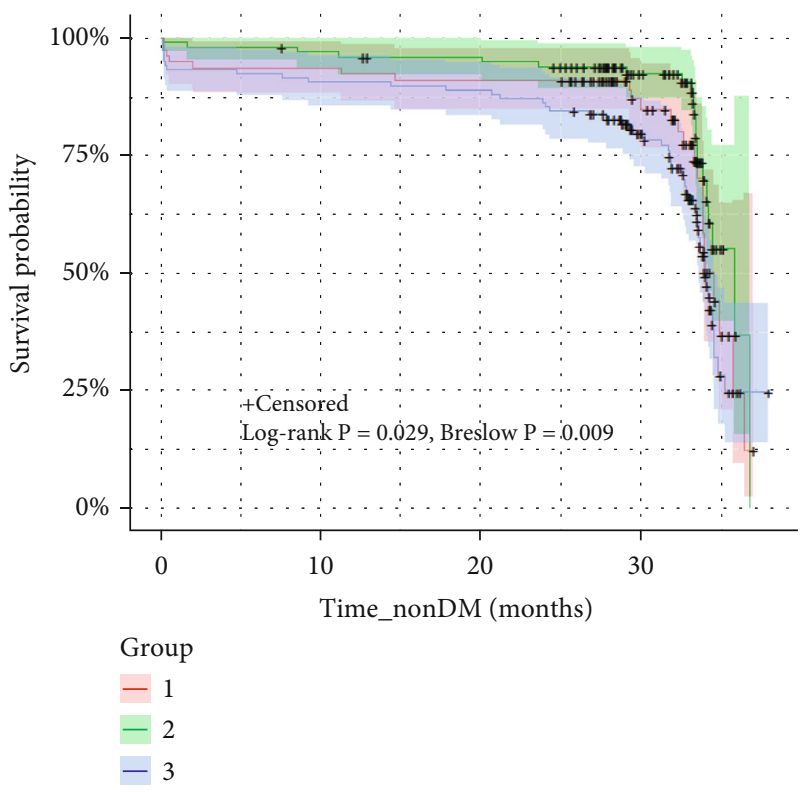

(b)

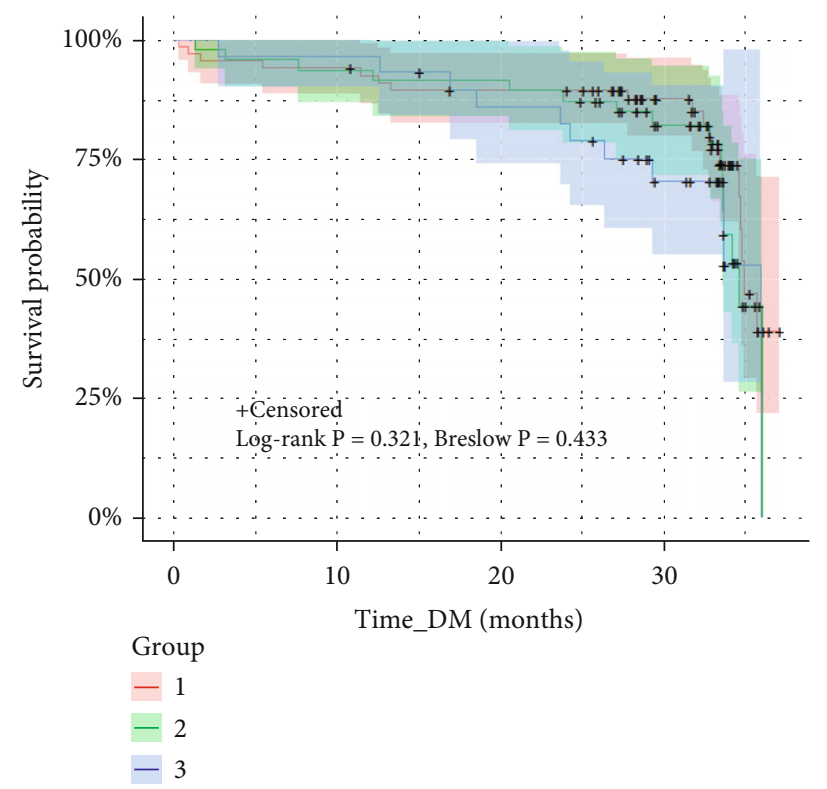

(c)

FIgure 2: Kaplan-Meier survival curves for MACEs. Cumulative Kaplan-Meier survival curves for MACEs in the (a) whole ACS patients undergoing stent implantation (b) without DM and (c) with DM by MCV level. Group 1, MCV-L; Group 2, MCV-M; Group 3, MCV-H.

normal distribution as confirmed by the KolmogorovSmirnov test. Unpaired $t$-test or one-way ANOVA or Mann-Whitney $U$ test was used to test the statistical significance of differences between the means or medians where appropriate. Categorical variables were expressed as frequency and proportions, and compared with the chi-square or Fisher exact test. Kaplan-Meier survival curves were constructed to assess the association of different MCV level groups with the occurrence of MACEs, and Cox regression models were constructed to calculate hazard ratios (HRs) and $95 \%$ confidence intervals (CIs) for MACEs. Landmark analyses were performed according to a landmark point of the 32 months. HRs and 95\% CIs were calculated separately for events occurring up to 32 months and those over the 32 nd month to the end of follow-up. Finally, the ROC curve was conducted.

All probability values were two-tailed. $P<0.05$ was considered statistically significant.

\section{Results}

3.1. Clinical Characteristics of Patients with and without DM. In the study, a total of 2153 patients were screened; 437 consecutive ACS patients who are undergoing stent implantation were analyzed, including 294 non-DM patients and 143 DM patients (Figure 1). During a median follow-up of 31.93 
TABLE 3: Cox regression analysis of MACEs in whole, nondiabetic, and diabetic patients.

\begin{tabular}{|c|c|c|c|c|c|c|}
\hline \multirow{2}{*}{ Variable } & \multicolumn{3}{|c|}{ Univariate analysis } & \multicolumn{3}{|c|}{ Multivariate analysis } \\
\hline & Hazard ratio $(95 \% \mathrm{CI})$ & SEM & $P$ value & Hazard ratio $(95 \% \mathrm{CI})$ & SEM & $P$ value \\
\hline \multicolumn{7}{|l|}{ Whole } \\
\hline MCV-L & Reference & & 0.032 & Reference & & 0.032 \\
\hline $\mathrm{MCV}-\mathrm{M}^{\mathrm{b}}$ & $0.899(0.577-1.401)$ & 0.226 & 0.638 & $1.159(0.662-2.028)$ & 0.286 & 0.606 \\
\hline $\mathrm{MCV}-\mathrm{H}^{\mathrm{b}}$ & $1.540(1.038-2.284)$ & 0.201 & 0.032 & $1.870(1.113-3.144)$ & 0.265 & 0.018 \\
\hline \multicolumn{7}{|l|}{ Non-DM } \\
\hline MCV-L & Reference & & 0.032 & Reference & & 0.032 \\
\hline $\mathrm{MCV}-\mathrm{M}^{\mathrm{b}}$ & $1.487(0.827-2.673)$ & 0.299 & 0.185 & $1.223(0.574-2.606)$ & 0.386 & 0.415 \\
\hline $\mathrm{MCV}-\mathrm{H}^{\mathrm{b}}$ & $1.963(1.180-3.265)$ & 0.260 & 0.009 & $2.089(1.077-4.501)$ & 0.338 & 0.029 \\
\hline \multicolumn{7}{|l|}{$\mathrm{DM}$} \\
\hline MCV-L & Reference & & 0.328 & & & \\
\hline $\mathrm{MCV}-\mathrm{M}^{\mathrm{b}}$ & $1.368(0.695-2.692)$ & 0.345 & 0.364 & & & \\
\hline $\mathrm{MCV}-\mathrm{H}^{\mathrm{b}}$ & $1.795(0.816-3.950)$ & 0.402 & 0.146 & & & \\
\hline
\end{tabular}

MACEs: major adverse cardiovascular events; MCV: mean corpuscular volume; DM, diabetes mellitus; CI: confidence interval; SEM: standard error of measurement. Adjusted for age, sex, body mass index, smoking, past myocardial infarction, hypertension, creatinine, creatine kinase isoenzymes, pro-B-type natriuretic peptide, and left ventricle ejection fraction. ${ }^{\mathrm{b}}$ Compared with MCV-L group.

months, 140 (32.03\%) were experienced MACEs. The basic characteristics of the whole, nondiabetic, and diabetic patients are presented in Table 1. Diabetic patients were elder, had lower MCV and HGB level compared with nondiabetic patients. Medications usage including aspirin (99.1\%), clopidogrel (97.3\%), ACEI/ARB (91.1\%), calcium channel blocker (21.7\%), $\beta$-blockers (88.1\%), and statin (98.2\%) are listed in Table 1.

3.2. Comparison of Basic Characteristics Stratified by MCV Tertiles. According to the above results, we found that the admission MCV level varied greatly between nondiabetic and diabetic patients. Therefore, all patients were segregated into three groups by tertiles of admission MCV level, and a comparison of the clinical data of these groups is shown in Table 2. The proportions of diabetes and HbA1c level were decreased as MCV level increased (all $P<0.05$ ). Moreover, the incidence of MACEs in the MCV-H group (41.8\%) was higher than that in the MCV-M (25.5\%) and MCV-L group (28.8\%, $P=0.007)$. No significant difference in other factors and medication use at discharge were observed amongst the three groups (all $P>0.05$ ).

3.3. MACEs Occurrence. Kaplan-Meier survival analysis demonstrated that higher admission MCV level was significantly associated with MACEs occurrence both in whole $(P=0.015$, log-rank test; $P=0.009$, Breslow test) and non-DM patients $(P=0.029$, log-rank test; $P=0.009$, Breslow test), but not in DM patients $(P=0.321$, log-rank test; $P=0.433$, Breslow test), as shown in Figure 2 .

We then used Cox regression model for further analysis as shown in Table 3. In univariate Cox regression analysis, we found MCV-H was significantly associated with an increased risk of MACEs in whole (HRs 1.540, 95\% CI 1.038-2.284, $P=0.032$ ) and non-DM patients (HRs 1.963, 95\% CI $1.180-3.265, P=0.009$ ) over a median of 31.93 months of follow-up. This relationship remained significant in whole (HRs 1.870, 95\% CI 1.113-3.144, $P=0.018$ ), espe- cially non-DM patients (HRs 2.089, 95\% CI 1.077-4.501, $P=0.029)$ after adjustment for age, sex, body mass index, smoking, past myocardial infarction, hypertension, creatinine, creatine kinase isoenzymes, pro-B-type natriuretic peptide, and left ventricle ejection fraction. However, MCV level was not associated with the risk of MACEs in DM patients.

Moreover, the whole and non-DM population were subjected to landmark survival analysis with a landmark point of 32 months. The cumulative survivals of MACEs were statistically different in whole and non-DM patients within 32 months, but with no significant differences in the time period over 32nd month to the end of follow-up (Figure 3). The results in Table 4 showed significantly higher MACEs in the MCV-H group than MCV-L group in the whole (HRs 2.979, 95\% CI1.331-6.671, $P=0.020)$, especially non-DM population (HRs 4.054, 95\% CI 1.192-13.789, $P=0.025$ ) within 32 months of follow-up but not in the later period (over 32nd month to the end of follow-up). The corresponding graphs were shown in Figure 4.

To further evaluate the predictive value for MACEs of admission MCV within 32 months, we applied the ROC curve analysis. As shown in Figure 5 and Supplementary Tables 1-2, the areas under curve for MACEs was 0.578 (95\% CI $0.506-0.651, P=0.026)$ in whole and $0.610(95 \%$ CI $0.517-0.703, P=0.017)$ in non-DM patients, whereas there was no association between MCV and MACEs in DM patients $(P>0.05)$. The optimal cut-off threshold for admission MCV in predicting MACEs following stent implantation was 94.25 (sensitivity/specificity: 56.6\%/62.7\%) in whole and 95.95 (sensitivity/specificity: 57.4\%/71.3\%) in non-DM populations.

\section{Discussion}

Previous studies showed that there was the high risk of allcause mortality in patients with ACS within one year after PCI [32]. Long-term glycometabolic disorder implied a high 


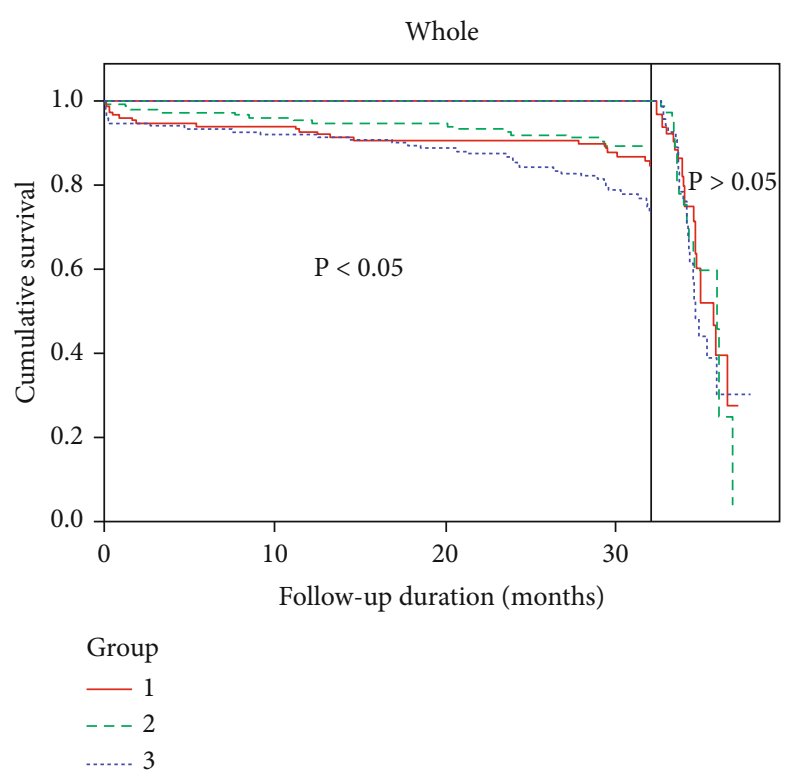

(a)

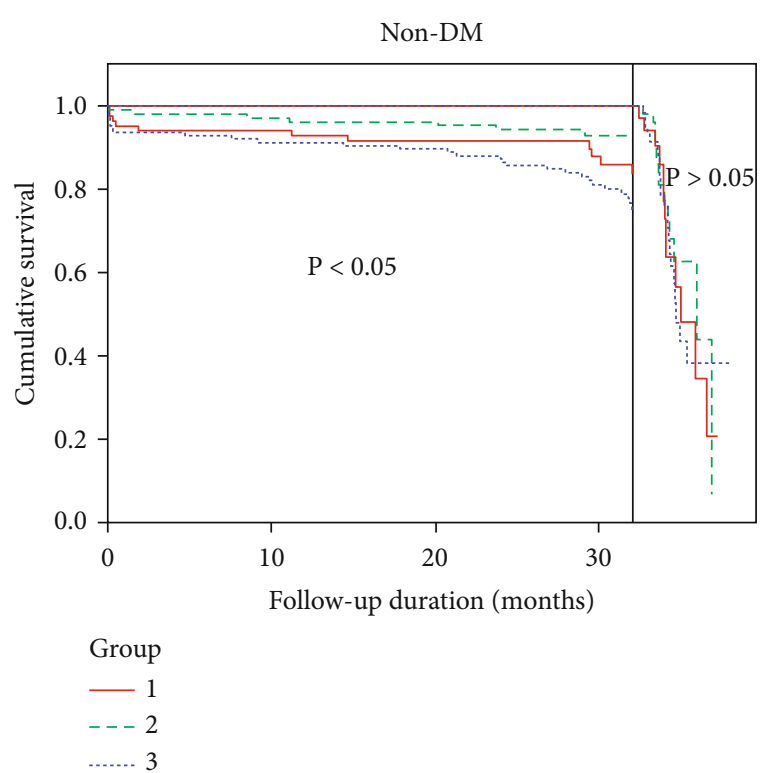

(b)

FIGURE 3: Landmark analysis of MACEs following stent implantation in whole and nondiabetic ACS patients. Kaplan-Meier survival curves (divided into two parts by the landmark point of 32 months) for MACEs (a) in the whole and (b) in non-DM population with ACS undergoing stent implantation by MCV level. Group 1, MCV-L; Group 2, MCV-M; Group 3, MCV-H.

TABLE 4: Landmark analysis of MACEs in whole and nondiabetic patients.

\begin{tabular}{|c|c|c|c|}
\hline Variable & Hazard ratio $(95 \% \mathrm{CI})$ & SEM & $P$ value \\
\hline \multicolumn{4}{|c|}{ Whole ( $\leq 32$ months) } \\
\hline MCV-L & Reference & & \\
\hline $\mathrm{MCV}-\mathrm{M}^{\mathrm{b}}$ & $1.359(0.536-3.447)$ & 0.478 & 0.518 \\
\hline $\mathrm{MCV}-\mathrm{H}^{\mathrm{b}}$ & $2.979(1.331-6.671)$ & 0.411 & 0.020 \\
\hline \multicolumn{4}{|c|}{ Whole ( $>32$ months to maximum follow-up) } \\
\hline MCV-L & Reference & & \\
\hline $\mathrm{MCV}-\mathrm{M}^{\mathrm{b}}$ & $0.933(0.447-1.945)$ & 0.375 & 0.852 \\
\hline $\mathrm{MCV}-\mathrm{H}^{\mathrm{b}}$ & $1.410(0.675-2.948)$ & 0.376 & 0.361 \\
\hline \multicolumn{4}{|c|}{ Non-DM ( $\leq 32$ months) } \\
\hline MCV-L & Reference & & \\
\hline $\mathrm{MCV}-\mathrm{M}^{\mathrm{b}}$ & $1.400(0.334-5.864)$ & 0.731 & 0.645 \\
\hline $\mathrm{MCV}-\mathrm{H}^{\mathrm{b}}$ & $4.054(1.192-13.789)$ & 0.625 & 0.025 \\
\hline \multicolumn{4}{|c|}{ Non-DM (>32 months to maximum follow-up) } \\
\hline MCV-L & Reference & & \\
\hline $\mathrm{MCV}-\mathrm{M}^{\mathrm{b}}$ & $1.119(0.420-2.985)$ & 0.501 & 0.822 \\
\hline $\mathrm{MCV}-\mathrm{H}^{\mathrm{b}}$ & $1.596(0.647-3.936)$ & 0.461 & 0.310 \\
\hline
\end{tabular}

ACS: acute coronary syndrome; MACEs: major adverse cardiovascular events; MCV: mean corpuscular volume; CI: confidence interval; SEM: standard error of measurement. ${ }^{\mathrm{b}}$ Compared with MCV-L group.

risk for cardiovascular disease and adverse outcomes $[33,34]$. Consequently, it is urgent to identify and manage risk factors of nondiabetic and diabetic patients in order to ameliorate adverse cardiovascular events. In the current study, we noted that MCV levels were higher in nondiabetic than diabetic ACS patients following stent implantation. Intriguingly, Kaplan-Meier survival analysis and Cox regression analysis demonstrated that the incidence of MACEs following stent implantation was significantly higher in MCV-H group com- pared with MCV-L group of whole, especially those nondiabetic populations but not diabetic populations over a median of 31.93 months of follow-up. Moreover, landmark analysis indicated that, in comparison to the MCV-L group, the increased risk of MCV-H group within 32 months was lost after 32nd month to the end of follow-up. Finally, the prognostic value of admission MCV within 32 months was further confirmed by ROC curve analysis in whole and nonDM patients. To the best of our knowledge, this is the first 


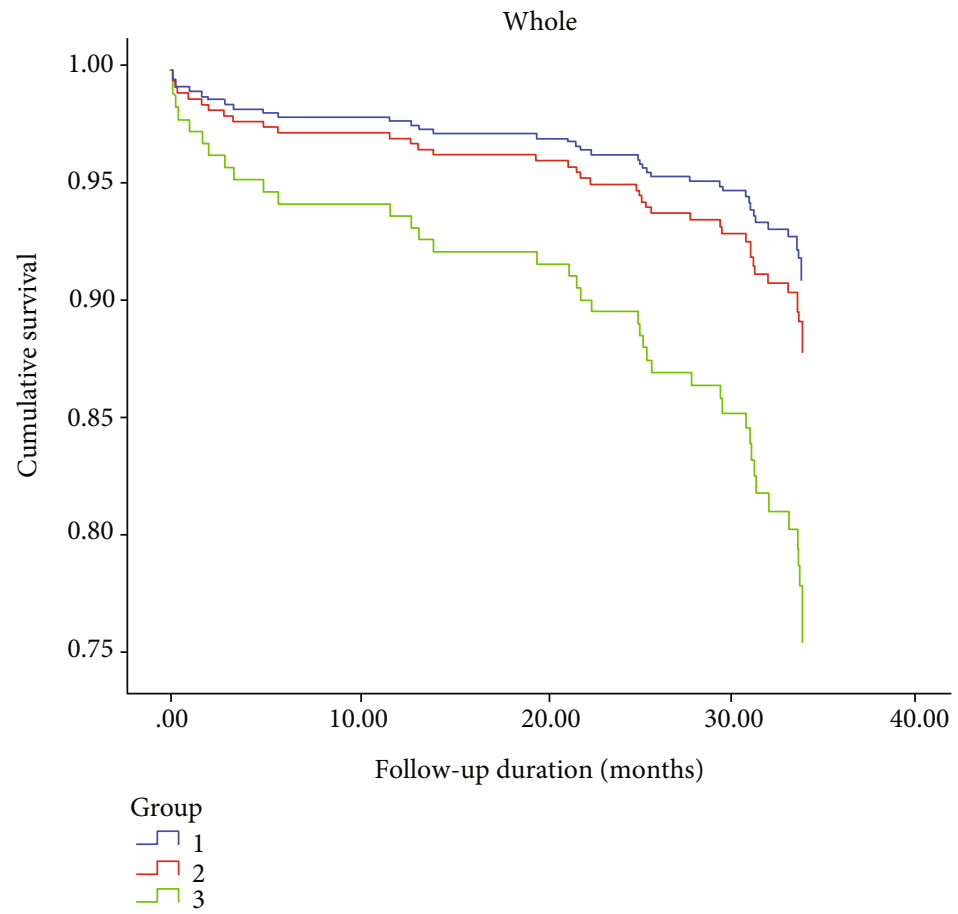

(a)

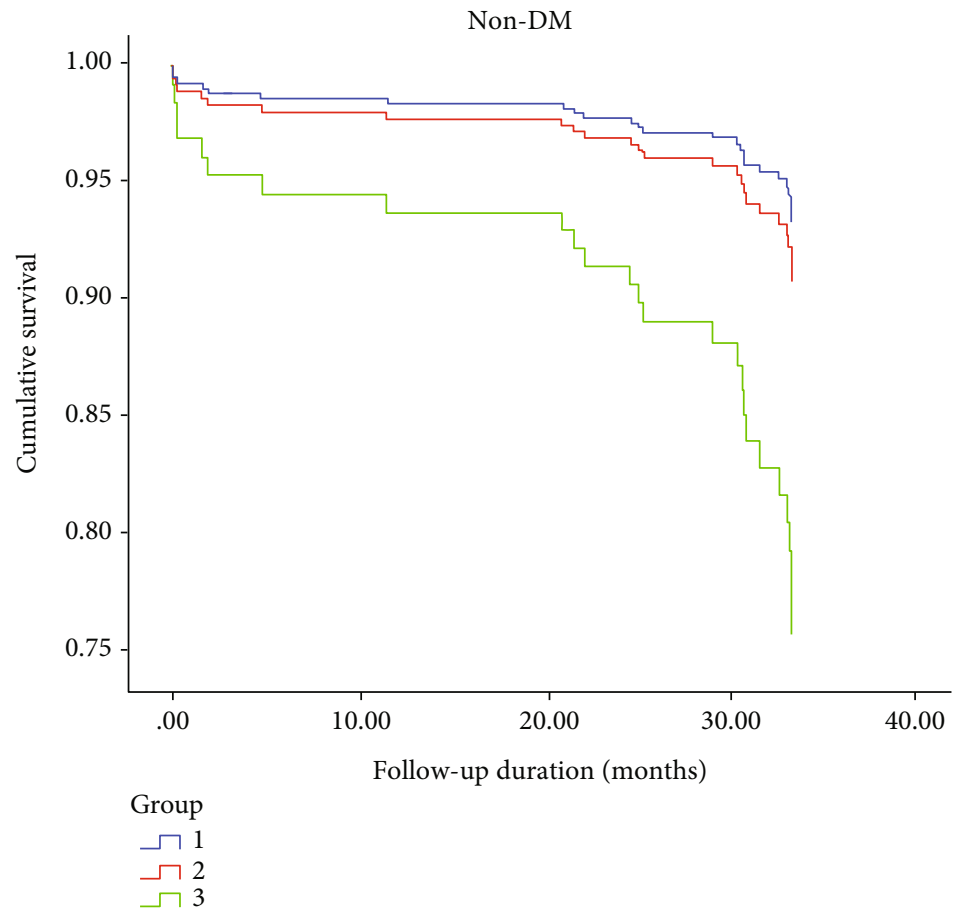

(b)

FIgURE 4: Cumulative survival curves for MACEs within 32 months. Cumulative survival curves for MACEs (a) in the whole and (b) non-DM population by MCV level within 32 months after adjust cardiovascular risk factors. Group 1, MCV-L; Group 2, MCV-M; Group 3, MCV-H.

study to focus on the relationship between admission MCV level and MACEs incidence following stent implantation in ACS patients with and without DM.

Chronic hyperglycemia induces ROS production, crosses the erythrocyte membrane, and oxidizes heme proteins, which have been shown to lead to the progressive loss of deformability of erythrocytes [35]. Diabetes can change hematological parameters that were related to hyperglycemia [14]. Previous studies observed that MCV was decreased in $\mathrm{DM}$ as compared to non-DM [17, 36]. Macrocytosis increased mortality, as well as main adverse cardiovascular and cerebrovascular events, among consecutive patients 


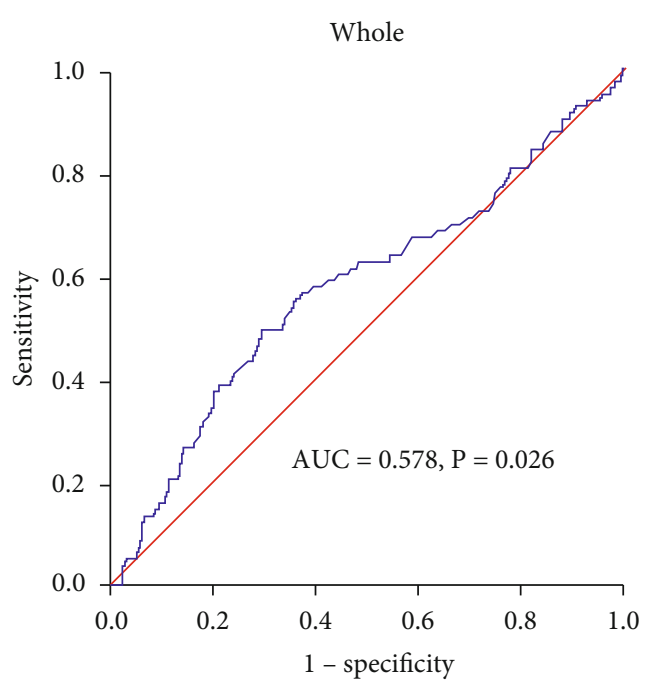

(a)

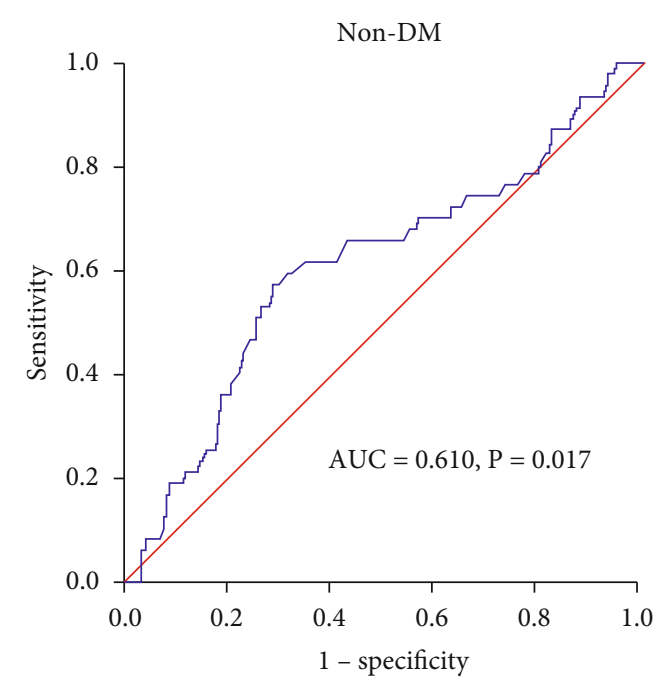

(b)

FIGURE 5: Receiver operating characteristic (ROC) curve analysis. ROC curve analysis on predictive value of admission MCV for MACEs following stent implantation (a) in whole ACS patients and (b) those without DM within 32 months.

who underwent PCI [37]. Increased MCV is associated with a higher incidence of subsequent cardiovascular event or death in nonanemic AMI patients [25]. However, the level of admission MCV and its role in MACEs occurrence of diabetic and nondiabetic ACS patients undergoing stent implantation receives not much attention. In the present study, the admission MCV level of non-DM patients was higher than that of DM patients. In addition, patients with MCV-H values exhibited high risk of MACEs in whole and non-DM ACS patients but not DM patients. Our study not only confirms the relationship between $\mathrm{MCV}$ and DM but also further assesses the association of MCV with MACEs incidence following stent implantation in ACS patients with or without DM. However, the conflicting evidence regarding MCV level and cardiovascular outcomes is reported. Stable CAD patients undergoing PCI in the 1st quartile who had the lowest MCV values exhibited a high risk of restenosis [22]. Obviously, the target population of this study are participants with stable $\mathrm{CAD}$, and their aim is to explore the association between $\mathrm{MCV}$ and incident restenosis; these details are very different from those of our study.

High MCV reduces the antioxidant function of red blood cells and is an independent prognosticator of impaired endothelial function measured via flow-mediated dilatation [24]. The direct mechanism of high MCV effect may be related to the increase of red blood cells resistance through microcirculation, which may lead to myocardial ischemia $[25,26]$. These factors may also be involved in the high occurrence of MACEs of MCV-H group in ACS patients undergoing stent implantation in this study. It is noteworthy that the significant difference of MACEs only exists in whole and nondiabetic patients in the present study might be due to chronic hyperglycemia and high HbAlc leads to low MCV level in general in DM patients.

The important implication of the present study is that landmark analyses revealed a better predictive value of admission MCV for MACEs after stent implantation in the first 32 months than in the subsequent period, and ROC curve confirmed its predictive value within 32 months in whole, especially non-DM populations, suggesting that admission MCV level cannot accurately reflect patients' outcomes with prolonged follow-up. To identify high-risk patients, current guidelines recommend a standardized approach involving validated scoring systems such as the Global Registry of Acute Coronary Events (GRACE) score $[38,39]$, which provides validated prognostic information for MACEs in ACS patients [40,41]. Therefore, combining admission MCV with the GRACE score may provide a more accurate risk estimation in ACS undergoing PCI which needs to be further explored.

The present study also has several limitations. Firstly, as an observational cohort study, there is inevitably some confounding bias, such as selection bias. Secondly, this research is a single-center study in a Chinese population, and the sample size is relatively small, especially in patients with DM; therefore, the comparisons of subgroups may lack power to detect significant differences for selected variables. Further multicenter and larger-scale surveys are required to confirm the results from this study and to elucidate the precise mechanisms. Moreover, the study only included ACS patients undergoing stent implantation, which suggests that the study results may not be extended to all ACS patients.

\section{Conclusions}

This study demonstrated that elevated admission MCV level was an important independent risk factor and had predictive value for MACEs following stent implantation in whole, especially those nondiabetic ACS patients. However, as the follow-up period increased, admission MCV lost its ability to predict MACEs. Measuring MCV, which is cheap and easy to obtain, might be helpful for a prognostic risk stratification 
of ACS patients undergoing stent implantation at a relatively short-term, especially those without DM.

\section{Abbreviations}

MCV: Mean corpuscular volume

ACS: $\quad$ Acute coronary syndromes

PCI: Percutaneous coronary intervention

DM: Diabetes mellitus

HbA1c: Hemoglobin A1c

CAD: Coronary artery disease

AMI: Acute myocardial infarction

UA: Unstable angina

STEMI: ST-segment elevation myocardial infarction

NSTEMI: Non-ST-segment elevation myocardial infarction

MACEs: Major adverse cardiovascular events

CABG: Percutaneous coronary intervention or coronary artery bypass grafting

HGB: Hemoglobin

hsCRP: High-sensitivity C-reactive protein

CKMB: Creatine kinase isoenzymes MB

pro-BNP: Pro-B-type natriuretic peptide

EF: $\quad$ Ejection fraction

TG: $\quad$ Triglyceride

LDL: Low-density lipoprotein cholesterol

HDL: High-density lipoprotein cholesterol

ACEI: Angiotensin-converting enzyme inhibition

ARB: Angiotensin receptor blocker

CCB: Calcium channel blocker

GRACE: Global registry of acute coronary events.

\section{Data Availability}

The datasets analyzed during the current study are available from the corresponding author on reasonable request.

\section{Ethical Approval}

This study was approved by the ethics committee of the First Affiliated Hospital of Xi'an Jiaotong University.

\section{Consent}

Written informed consent was obtained from all study participants.

\section{Conflicts of Interest}

The authors declare that they have no conflicts of interest.

\section{Authors' Contributions}

ZY conceived and designed the study; YW and LC contributed to the design; LC performed the study; JL, LZ, RL, XB, LW, WL, BL, and JZ help to the study; LC analyzed the data and draft the paper; YW contributed to the drafting. All authors read and critically revised the final manuscript. All authors read and approved the final manuscript.

\section{Acknowledgments}

This study was supported by the National Natural Science Foundation of China (Nos. 81822005, 91639301), Key Project of Research and Development Plan of Shaanxi Province (2017ZDCXL-SF-02-04-01), and National Key R\&D Program of China (2018YFC1311505).

\section{Supplementary Materials}

Supplementary Table 1: the coordinate of ROC Curve for admission MCV associated with MACEs in non-DM populations within 32 months. Supplementary Table 2: the coordinate of ROC Curve for admission MCV associated with MACEs in whole populations within 32 months. (Supplementary Materials)

\section{References}

[1] B. Unal, K. Sözmen, H. Arık et al., "Explaining the decline in coronary heart disease mortality in Turkey between 1995 and 2008," BMC Public Health, vol. 13, no. 1, article 1135, 2013.

[2] C. P. Cannon, S. Shah, H. M. Dansky et al., "Safety of anacetrapib in patients with or at high risk for coronary heart disease," The New England Journal of Medicine, vol. 363, no. 25, pp. 2406-2415, 2010.

[3] T. S. Polonsky, R. McClelland, N. W. Jorgensen et al., "Coronary artery calcium score and risk classification for coronary heart disease prediction," Journal of the American Medical Association, vol. 303, no. 16, pp. 1610-1616, 2010.

[4] N. D. Wong, "Epidemiological studies of CHD and the evolution of preventive cardiology," Nature Reviews Cardiology, vol. 11, no. 5, pp. 276-289, 2014.

[5] C. J. L. Murray, R. M. Barber, K. J. Foreman et al., "Global, regional, and national disability-adjusted life years (DALYs) for 306 diseases and injuries and healthy life expectancy (HALE) for 188 countries, 1990-2013: quantifying the epidemiological transition," The Lancet, vol. 386, no. 10009, pp. 2145-2191, 2015.

[6] H. F. AlFaleh, K. F. AlHabib, T. Kashour et al., "Short-term and long-term adverse cardiovascular events across the glycaemic spectrum in patients with acute coronary syndrome," Coronary Artery Disease, vol. 25, no. 4, pp. 330-338, 2014.

[7] Q. Mao, D. Zhou, Y. Li, Y. Wang, S. C. Xu, and X. H. Zhao, "The triglyceride-glucose index predicts coronary artery disease severity and cardiovascular outcomes in patients with non-ST-segment elevation acute coronary syndrome," Disease Markers, vol. 2019, Article ID 6891537, 11 pages, 2019.

[8] A. Cassar, D. R. Holmes Jr., C. S. Rihal, and B. J. Gersh, "Chronic coronary artery disease: diagnosis and management," Mayo Clinic Proceedings, vol. 84, no. 12, pp. 11301146, 2009.

[9] A. Bagnall and I. Spyridopoulos, "The evidence base for revascularisation of chronic total occlusions," Current Cardiology Reviews, vol. 10, no. 2, pp. 88-98, 2014.

[10] G. De Luca, M. Verdoia, and H. Suryapranata, "Benefits from intracoronary as compared to intravenous abciximab administration for STEMI patients undergoing primary angioplasty: A meta- analysis of 8 randomized trials," Atherosclerosis, vol. 222, no. 2, pp. 426-433, 2012. 
[11] Z. W. Zhao, Y. W. Xu, S. M. Li et al., "Baseline serum sLOX-1 concentrations are associated with 2-year major adverse cardiovascular and cerebrovascular events in patients after percutaneous coronary intervention," Disease Markers, vol. 2019, Article ID 4925767, 8 pages, 2019.

[12] G. De Luca, M. T. Dirksen, C. Spaulding et al., "Impact of diabetes on long-term outcome after primary angioplasty: insights from the DESERT cooperation," Diabetes Care, vol. 36, no. 4, pp. 1020-1025, 2013.

[13] Z. Dong, Z. Liang, M. Guo, S. Hu, Z. Shen, and X. Hai, "The association between plasma levels of trimethylamine $\mathrm{N}$-oxide and the risk of coronary heart disease in Chinese patients with or without type 2 diabetes mellitus," Disease Markers, vol. 2018, Article ID 1578320, 7 pages, 2018.

[14] M. C. Thomas, R. J. MacIsaac, C. Tsalamandris, D. Power, and G. Jerums, "Unrecognized anemia in patients with Diabetes: A cross-sectional survey," Diabetes Care, vol. 26, no. 4, pp. 11641169, 2003.

[15] A. Tefferi, "Anemia in adults: a contemporary approach to diagnosis," Mayo Clinic Proceedings, vol. 78, no. 10, pp. 1274-1280, 2003.

[16] M. J. Cascio and T. G. DeLoughery, "Anemia: evaluation and diagnostic tests," The Medical Clinics of North America, vol. 101, no. 2, pp. 263-284, 2017.

[17] P. S. Hardikar, S. M. Joshi, D. S. Bhat et al., "Spuriously high prevalence of prediabetes diagnosed by $\mathrm{HbAlc}$ in young Indians partly explained by hematological factors and iron deficiency anemia," Diabetes Care, vol. 35, no. 4, pp. 797802, 2012.

[18] P. E. Stevens, "Anaemia, diabetes and chronic kidney disease: where are we now?," Journal of Renal Care, vol. 38, Supplement 1, pp. 67-77, 2012.

[19] E. Stolz, J. M. Valdueza, M. Grebe et al., "Anemia as a risk factor for cerebral venous thrombosis? An old hypothesis revisited. Results of a prospective study," Journal of Neurology, vol. 254, no. 6, pp. 729-734, 2007.

[20] T. Ueda, R. Kawakami, M. Horii et al., "High mean corpuscular volume is a new indicator of prognosis in acute decompensated heart failure," Circulation Journal, vol. 77, no. 11, pp. 2766-2771, 2013.

[21] Y. L. Zhan, B. Zou, T. Kang, L. B. Xiong, J. Zou, and Y. F. Wei, "Multiplicative interaction between mean corpuscular volume and red cell distribution width with target organ damage in hypertensive patients," Journal of Clinical Laboratory Analysis, vol. 31, no. 5, article e22082, 2017.

[22] L. Sun, C. Zhang, Y. Ju et al., "Mean corpuscular volume predicts in-stent restenosis risk for stable coronary artery disease patients receiving elective percutaneous coronary intervention,” Medical Science Monitor, vol. 25, pp. 3976-3982, 2019.

[23] T. H. Wu, J. C. Y. Fann, S. L. S. Chen et al., "Gradient Relationship between Increased Mean Corpuscular Volume and Mortality Associated with Cerebral Ischemic Stroke and Ischemic Heart Disease: A Longitudinal Study on 66,294 Taiwanese," Scientific Reports, vol. 8, no. 1, article 16517, 2018.

[24] Y. Solak, M. I. Yilmaz, M. Saglam et al., "Mean corpuscular volume is associated with endothelial dysfunction and predicts composite cardiovascular events in patients with chronic kidney disease," Nephrology, vol. 18, no. 11, pp. 728-735, 2013.

[25] P. Franczuk, M. Kaczorowski, K. Kucharska et al., "Could an analysis of mean corpuscular volume help to improve risk stratification in non-anemic patients with acute myocardial infarction?," Cardiology Journal, vol. 22, no. 4, pp. 421-427, 2015.

[26] K. Kubota, J.'. I. Tamura, T. Shirakura et al., "The behaviour of red cells in narrow tubes in vitro as a model of the microcirculation," British Journal of Haematology, vol. 94, no. 2, pp. 266272, 1996.

[27] Writing Committee Members, G. N. Levine, E. R. Bates et al., "2011 ACCF/AHA/SCAI guideline for percutaneous coronary Intervention: a report of the American College of Cardiology Foundation/American Heart Association Task Force on Practice Guidelines and the Society for Cardiovascular Angiography and Interventions," Circulation, vol. 124, no. 23, pp. e574-e651, 2011.

[28] T. Palmerini, P. Genereux, A. Caixeta et al., "Prognostic Value of the SYNTAX Score in Patients With Acute Coronary Syndromes Undergoing Percutaneous Coronary Intervention: Analysis From the ACUITY (Acute Catheterization and Urgent Intervention Triage StrategY) Trial," Journal of the American College of Cardiology, vol. 57, no. 24, pp. 23892397, 2011.

[29] J. Cohen, Statistical Power Analysis for the Behavioral Sciences, Lawrence Erlbaum Associates, Hillsdale, New Jersey, 1988.

[30] Y. Handelsman, J. I. Mechanick, L. Blonde et al., “American association of clinical endocrinologists medical guidelines for clinical practice for developing a diabetes mellitus comprehensive care plan," Endocrine Practice, vol. 17, Supplement 2, pp. 1-53, 2011.

[31] I. Ferreira-González, G. Permanyer-Miralda, A. Domingo-Salvany et al., "Problems with use of composite end points in cardiovascular trials: systematic review of randomised controlled trials,” BMJ, vol. 334, no. 7597, p. 786, 2007.

[32] V. Veeranna, S. K. Zalawadiya, A. Niraj et al., "Homocysteine and reclassification of cardiovascular disease risk," Journal of the American College of Cardiology, vol. 58, no. 10, pp. 10251033, 2011.

[33] D. O. Kang, H. S. Seo, B. G. Choi et al., "Absolute change in fasting plasma glucose over 12 months is associated with 2year and 5-year major adverse cardiovascular events in patients with drug- eluting stent implants," International Journal of Cardiology, vol. 179, pp. 146-152, 2015.

[34] B. Lin, N. Koibuchi, Y. Hasegawa et al., "Glycemic control with empagliflozin, a novel selective SGLT2 inhibitor, ameliorates cardiovascular injury and cognitive dysfunction in obese and type 2 diabetic mice," Cardiovascular Diabetology, vol. 13, no. 1, p. 148, 2014.

[35] S. Jagadish, M. Hemshekhar, S. K. NaveenKumar et al., "Novel oxolane derivative DMTD mitigates high glucose-induced erythrocyte apoptosis by regulating oxidative stress," Toxicology and Applied Pharmacology, vol. 334, pp. 167-179, 2017.

[36] A. Ludidi, M. C. Baloyi, A. Khathi, N. H. Sibiya, and P. S. Ngubane, "The effects of Momordica balsamina methanolic extract on haematological function in streptozotocin-induced diabetic rats: Effects on selected markers," Biomedicine \& Pharmacotherapy, vol. 116, article 108925, 2019.

[37] M. Myojo, H. Iwata, T. Kohro et al., "Prognostic implication of macrocytosis on adverse outcomes after coronary intervention," Atherosclerosis, vol. 221, no. 1, pp. 148-153, 2012.

[38] C. W. Hamm, J. P. Bassand, S. Agewall et al., "ESC guidelines for the management of acute coronary syndromes in patients presenting without persistent ST-segment elevation. The task force for the management of acute coronary syndromes (ACS) in patients presenting without persistent ST-segment 
elevation of the European Society of Cardiology (ESC)," Giornale Italiano Di Cardiologia, vol. 13, no. 3, pp. 171-228, 2012.

[39] 2007 Writing Committee Members, J. L. Anderson, C. D. Adams et al., "2012 ACCF/AHA focused update incorporated into the ACCF/AHA 2007 guidelines for the management of patients with unstable angina/non-ST-elevation myocardial Infarction," Circulation, vol. 127, no. 23, pp. e663-e828, 2013.

[40] E. W. Tang, C. K. Wong, and P. Herbison, "Global registry of acute coronary events (GRACE) hospital discharge risk score accurately predicts long-term mortality post acute coronary syndrome," American Heart Journal, vol. 153, no. 1, pp. 2935, 2007.

[41] K. A. Eagle, M. J. Lim, O. H. Dabbous et al., "A validated prediction model for all forms of acute coronary Syndrome," Journal of the American Medical Association, vol. 291, no. 22, pp. 2727-2733, 2004. 